\title{
3D DOPs for Positioning Applications Using Range Measurements
}

\author{
Binghao Li ${ }^{1}$, Andrew G. Dempster ${ }^{1}$, Jian Wang ${ }^{2}$ \\ ${ }^{1}$ School of Surveying and Spatial Information Systems, University of New South Wales, Sydney, Australia \\ ${ }^{2}$ School of Environment and Spatial Informatics, China University of Mining \& Technology, Xuzhou, China \\ E-mail: \{binghao.li,a.dempster\}@unsw.edu.au,wjian@cumt.edu.cn \\ Received August 22, 2011; revised September 20, 2011; accepted September 30, 2011
}

\begin{abstract}
For terrestrial positioning, some applications require three dimensional coordinates. The Dilution of precisions (DOPs) for position systems using range measurement is reviewed and the average values of DOPs for different deployments of base station geometries are examined. It is shown that to obtain the lowest DOPs, the base stations for different types of positioning systems need to be deployed differently. Changing the $N$-sided regular polygon to an $(N-1)$-sided polygon with one base station in the centre of the polygon can decrease the value of DOP in general for a pseudorange time of arrival (TOA) system but not for an absolute range TOA system. The height of the base station in the centre can also change the DOP significantly. The finding can be used to optimize the deployment of the base stations for range measurement positioning systems.
\end{abstract}

Keywords: Positioning, 3D DOP, TOA, TDOA

\section{Introduction}

Using range measurements for positioning is the method most popular in satellite navigation systems. The typical example is the Global Positioning System (GPS) which uses the Time of Arrival (TOA) to estimate the receiver's position [1]. Similar examples can be found in terrestrial positioning systems [2-5]. Since, in these systems, the clock error in the receiver is unknown, pseudorange rather than the absolute range is measured. If the transmitters and receiver are well synchronized or a round trip time (RTT) can be obtained, the absolute range measurement can be used for positioning [6]. To distinguish these two technologies, the former is called pseudorange processing and the latter is named absolute range processing in this paper. Time Difference of Arrival (TDOA) is also applied in many positioning systems such as the widely used Loran-C and Omega [7], primarily used before the satellite positioning era, and the global cellular network [8]. A TDOA system is also known as a hyperbolic multilateration system as it relies on the fact that all the points where the difference in the TOA radio signals from different stations is constant, its "line of position", forms a hyperbola.

No matter what range measurement is used, to obtain an accurate user position requires a good geometrical distribution of the base stations or beacons. Dilution of precision (DOP) is used to describe the effect of geometry on the relationship between measurement error and position determination error. DOP has been well investigated for Global Navigation Satellite Systems (GNSS) [9] [10-12]. However, originally it was discussed in hyperbolic multilateration systems $[13,14]$. In terrestrial positioning systems, the 2D coordinate can be of more of interest $[15,16]$. But in some applications such as machine guidance, warehouse management and emergency services, 3D coordinates are often required. As an example, fire fighters enter a building that they are not familiar with. In an environment full of smoke, with poor visibility and unknown dangers, 3D position is needed to navigate to the correct floor. Base stations can be installed on the fire engines and deployed quickly around the building in fire to facilitate this requirement [3].

Several potential positioning systems for the emergency applications have been reported, such as a mobile wireless localisation network known as WASP (Wireless Ad-hoc Self Positioning) which can achieve better than half-metre accuracy in non-line-of-sight (NLOS) environments. The Worcester Polytechnic Institute (WPI) Precision Personnel Locator (PPL) project demonstrated 
better than $1 \mathrm{~m}$ accuracy in high multipath environments [3]; and a Ultra Wide Band (UWB) system was reported to have achieved $15 \mathrm{~cm}$ location accuracy in an open environment [17].

How to deploy the base stations to achieve a better DOP for the area of interest must be investigated as the DOP value has a great impact on the positioning accuracy. In the following sections, the theoretical issues of DOP are reviewed first in Section 2, and then the deployment of the base stations is discussed in Section 3. Finally, the conclusion is given.

\section{Dilution of Precession}

In 1975 Lee published the first paper [14] to discuss Geometric DOP (GDOP) of hyperbolic multilateration systems. GDOP is defined as the ratio of the root mean square (rms) position error to the rms ranging error. Although only TDOA was discussed, the fundamental work can be easily applied to absolute range or pseudorange systems. Similarly, Position DOP (PDOP), Horizontal DOP, Vertical DOP (VDOP) can be defined.

A range measurement can be expressed as:

$$
L=f(x, y, z)
$$

where $L$ is a measured value, and $x, y$ and $z$ are unknown parameters (the coordinate of the user). To linearize the equation, Taylor's theorem is normally applied

$$
\begin{aligned}
L= & f\left(x_{0}, y_{0}, z_{0}\right)+\frac{(\partial L / \partial x)_{0} \mathrm{~d} x}{1 !}+\frac{(\partial L / \partial y)_{0} \mathrm{~d} y}{1 !} \\
& +\frac{(\partial L / \partial z)_{0} \mathrm{~d} z}{1 !}+\cdots+\frac{\left(\partial^{n} L / \partial x^{n}\right)_{0} \mathrm{~d} x^{n}}{n !} \\
& +\frac{\left(\partial^{n} L / \partial y^{n}\right)_{0} \mathrm{~d} y^{n}}{n !}+\frac{\left(\partial^{n} L / \partial x z^{n}\right)_{0} \mathrm{~d} z^{n}}{n !}+R_{n}
\end{aligned}
$$

Hence

$$
\begin{aligned}
& f(x, y, z)-f\left(x_{0}, y_{0}, z_{0}\right) \\
= & (\partial L / \partial x)_{0} \mathrm{~d} x+(\partial L / \partial y)_{0} \mathrm{~d} y+(\partial L / \partial z)_{0} \mathrm{~d} z
\end{aligned}
$$

Assume there are $n$ observations, matrix notation can be used:

$$
\boldsymbol{H} \Delta x=\Delta r
$$

where $\Delta x$ is the vector of offset of the true position of the user from the linearization point, $\Delta r$ is the vector offset of the true range to the range values corresponding to the linearization point, and $\boldsymbol{H}$ can be present as

$$
\boldsymbol{H}=\left[\begin{array}{ccc}
\left(\partial L_{1} / \partial x\right)_{0} & \left(\partial L_{1} / \partial y\right)_{0} & \cdots \\
\left(\partial L_{2} / \partial x\right)_{0} & \left(\partial L_{2} / \partial y\right)_{0} & \cdots \\
\vdots & \vdots & \vdots \\
\left(\partial L_{n} / \partial x\right)_{0} & \left(\partial L_{n} / \partial y\right)_{0} & \cdots
\end{array}\right]
$$

\subsection{Absolute Range Processing}

If absolute range measurements are available (the user is synchronized with the base stations or the round trip of the signal is available), the range from the user to the $i$ th base station is:

$$
R_{i}=\sqrt{\left(x-x_{i}\right)^{2}+\left(y-y_{i}\right)^{2}+\left(z-z_{i}\right)^{2}}
$$

where $\left(x_{i}, y_{i}, z_{i}\right)$ is the coordinate of $i$ th base station.

There are $n$ measurements

$$
R=\left[\begin{array}{lll}
r_{1} & \cdots & r_{n}
\end{array}\right]^{T}
$$

The vector of unknowns is

$$
\boldsymbol{X}=\left[\begin{array}{lll}
x & y & z
\end{array}\right]^{T}
$$

Assuming the errors in the measurements are random, independent, have zero mean and have an identical rms $\sigma_{r}$, the error covariance matrix is

$$
\boldsymbol{Q}=\sigma_{r}^{2} I
$$

The $\boldsymbol{H}$ matrix in Equation (4) can be expressed as

$$
\boldsymbol{H}=\left[\begin{array}{ccc}
\frac{x-x_{1}}{R_{1}} & \frac{y-y_{1}}{R_{1}} & \frac{z-z_{1}}{R_{1}} \\
\vdots & \vdots & \vdots \\
\frac{x-x_{n}}{R_{n}} & \frac{y-y_{n}}{R_{n}} & \frac{z-z_{n}}{R_{n}}
\end{array}\right]
$$

When least squares is used $(n \geq 3)$

$$
\Delta x=\left(H^{T} \boldsymbol{H}\right)^{-1} H^{T} \Delta r
$$

It can also be expressed as

$$
\left(x_{T}-x_{L}+\mathrm{d} x\right)=\left(H^{T} \boldsymbol{H}\right)^{-1} H^{T}\left(\boldsymbol{r}_{\boldsymbol{T}}-\boldsymbol{r}_{\boldsymbol{L}}+\mathrm{d} r\right)
$$

where $x_{T}$ is the error-free position, $x_{L}$ is the position defined as the linearization point and $\mathrm{d} x$ is the position error. $\boldsymbol{r}_{T}$ represents the vector of true range values, $\boldsymbol{r}_{\boldsymbol{L}}$ is the vector of range values computed at the linearization point and $\mathrm{d} r$ is the range measurement error. Hence

$$
\mathrm{d} x=\left(H^{T} \boldsymbol{H}\right)^{-1} H^{T} \mathrm{~d} r
$$

DOP is defined as

$$
\mathrm{DOP}=\frac{\sigma_{x}}{\sigma_{r}}
$$

since

$$
\begin{aligned}
\operatorname{cov}(\mathrm{d} x) & =E\left(\mathrm{~d} x \mathrm{~d} x^{T}\right) \\
& =E\left[\left(H^{T} \boldsymbol{H}\right)^{-1} H^{T} \mathrm{~d} r \mathrm{~d} r^{T} \boldsymbol{H}\left(H^{T} \boldsymbol{H}\right)^{-1}\right] \\
& =\left(H^{T} \boldsymbol{H}\right)^{-1} H^{T} \operatorname{cov}(\mathrm{d} r) \boldsymbol{H}\left(H^{T} \boldsymbol{H}\right)^{-1} \\
& =\left(H^{T} \boldsymbol{H}\right)^{-1} \sigma_{r}{ }^{2}
\end{aligned}
$$


where $\operatorname{cov}(\mathrm{d} r)=I \sigma_{r}^{2}$

$$
\begin{array}{r}
\mathrm{HDOP}=\frac{\sqrt{\sigma_{x}^{2}+\sigma_{y}^{2}}}{\sigma_{r}}=\sqrt{\left(\left(H^{T} \boldsymbol{H}\right)^{-1}\right)_{1,1}+\left(\left(H^{T} \boldsymbol{H}\right)^{-1}\right)_{2,2}} \\
\mathrm{VDOP}=\frac{\sqrt{\sigma_{z}^{2}}}{\sigma_{r}}=\sqrt{\left(\left(H^{T} \boldsymbol{H}\right)^{-1}\right)_{3,3}} \\
\mathrm{PDOP}=\frac{\sqrt{\sigma_{x}^{2}+\sigma_{y}^{2}+\sigma_{z}^{2}}}{\sigma_{r}} \\
=\sqrt{\left(\left(H^{T} \boldsymbol{H}\right)^{-1}\right)_{1,1}+\left(\left(H^{T} \boldsymbol{H}\right)^{-1}\right)_{2,2}+\left(\left(H^{T} \boldsymbol{H}\right)^{-1}\right)_{3,3}} \\
\mathrm{GDOP}=\sqrt{\operatorname{trace}\left(\left(H^{T} \boldsymbol{H}\right)^{-1}\right)}
\end{array}
$$

In this case, PDOP is the same as GDOP. Obviously, when range measurements are used, DOP is unitless, whereas in angle of arrival, there are units of meters [18].

\subsection{Pseudorange Processing}

When pseudorange measurements are used, there is one more unknown - the receiver clock error. Hence the range from the user to the $i$ th base station is

$$
R_{i}=\sqrt{\left(x-x_{i}\right)^{2}+\left(y-y_{i}\right)^{2}+\left(z-z_{i}\right)^{2}}+\Delta t
$$

Similarly to the case for absolute range, there are $n$ measurements but the vector of unknowns is slightly different

$$
\boldsymbol{X}=\left[\begin{array}{llll}
x & y & z & \Delta t
\end{array}\right]^{T}
$$

Then the $\boldsymbol{H}$ matrix can be expressed as

$$
\boldsymbol{H}=\left[\begin{array}{cccc}
\frac{x-x_{1}}{R_{1}} & \frac{y-y_{1}}{R_{1}} & \frac{z-z_{1}}{R_{1}} & 1 \\
\vdots & \vdots & \vdots & \vdots \\
\frac{x-x_{n}}{R_{n}} & \frac{y-y_{n}}{R_{n}} & \frac{z-z_{n}}{R_{n}} & 1
\end{array}\right]
$$

All the $D O P$ s are similar except the $G D O P$ since there is one more diagonal element in $\left(H^{T} \boldsymbol{H}\right)^{-1}$ which is TDOP

$$
\mathrm{TDOP}=\frac{\sqrt{\sigma_{t}^{2}}}{\sigma_{r}}=\sqrt{\left(\left(H^{T} \boldsymbol{H}\right)^{-1}\right)_{4,4}}
$$

\subsection{TDOA}

The TDOA measurement can be presented as

$$
\begin{aligned}
\Delta R_{i}= & \sqrt{\left(x-x_{1}\right)^{2}+\left(y-y_{1}\right)^{2}+\left(z-z_{1}\right)^{2}} \\
& -\sqrt{\left(x-x_{i}\right)^{2}+\left(y-y_{i}\right)^{2}+\left(z-z_{i}\right)^{2}} \quad i=2,3, \cdots
\end{aligned}
$$

The measurements are

$$
\Delta R=\left[\begin{array}{lll}
\Delta r_{2} & \cdots & \Delta r_{n}
\end{array}\right]^{T}
$$

The vector of unknowns is the same as Equation (7), but the $\boldsymbol{H}$ matrix is different

$$
\boldsymbol{H}=\left[\begin{array}{cccc}
\frac{x-x_{1}}{R_{1}}-\frac{x-x_{2}}{R_{2}} & \frac{y-y_{1}}{R_{1}}-\frac{y-y_{2}}{R_{2}} & \frac{z-z_{1}}{R_{1}}-\frac{z-z_{2}}{R_{2}} \\
\vdots & \vdots & \vdots \\
\frac{x-x_{1}}{R_{1}}-\frac{x-x_{n}}{R_{n}} & \frac{y-y_{1}}{R_{1}}-\frac{y-y_{n}}{R_{n}} & \frac{z-z_{1}}{R_{1}}-\frac{z-z_{n}}{R_{n}}
\end{array}\right]
$$

And the error covariance matrix is no longer an identity matrix

$$
\boldsymbol{Q}=\sigma_{r}^{2}\left(\begin{array}{cccc}
2 & 1 & \cdots & 1 \\
1 & 2 & \cdots & 1 \\
\vdots & \vdots & \vdots & \vdots \\
1 & 1 & 1 & 2
\end{array}\right)
$$

The DOPs are expressed in the same way as equations (15) to (18). GDOP and PDOP are identical.

\section{3D DOPS Associated with Different Deployments of Base Stations}

In real applications, there are many restrictions on the deployment of the base stations. To discuss the 3D DOPs, the scenarios are simplified as follows:

The projection of the base stations on the $x-y$ plane forms an $N$-sided regular polygon where the vertices are the locations of the projection of the base stations. The radius of the circumscribed circle is $a$ (see Figure 1). The heights of the base stations can be configured to set up different scenarios. To make a fair comparison of the scenarios with different numbers of base stations, the area of interest is that inside the circumscribed circle on the $x-y$ plane (the shadowed area in Figure 1). The average DOP in this area is calculated. The square area outside the circumscribed circle is used to show the mesh plot of GDOP results. It is reported in [19] that at the centre of a regular polygon the lowest "GDOP", more precisely PDOP (note the GDOP in pseudorange processing includes TDOP element but otherwise is the same as PDOP), can be achieve in 2D scenarios $(2 / \sqrt{N})$ when absolute range and pseudorange are used for positioning. In [13], it is shown that for TDOA systems the minimum GDOP is $2 / \sqrt{N}$ and $3 / \sqrt{N}$ ( $N$ is the num 


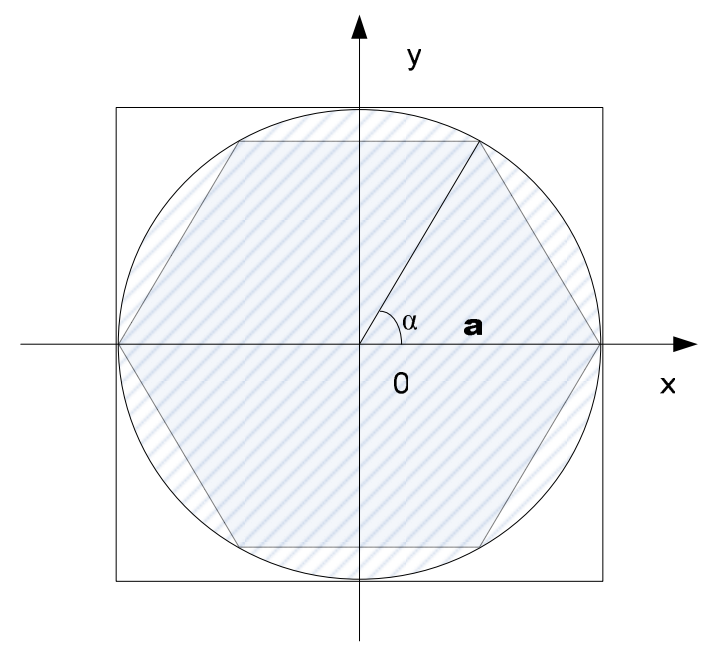

Figure 1. Six-sided regular polygon and the area of interest to calculate the DOPs.

ber of transmitters) in 2D and 3D scenarios respectively. Shin and Sung [20] prove that for TDOA and TOA processing

$$
\mathrm{PDOP}_{\mathrm{TDOA}}=\mathrm{PDOP}_{\mathrm{TOA}}
$$

and the equality also holds for HDOP and VDOP. This conclusion is consistent with the results in $[13,14,19]$. It has also been confirmed by calculating the average DOPs for different scenarios. Hence further comparisons are only for absolute range processing and pseudorange processing.

Obviously, if the base stations are all in the same plane as that of the user, e.g. the $x-y$ plane, DOPs are infinite. The height of the base stations should be different from the user. If the maximum height of the base stations is restricted by the application to 0.1 times of $a$ (for example, the base stations on a fire engine cannot be lifted more than $5 \mathrm{~m}$, say, but the distance between two fire engines can be easily more than $50 \mathrm{~m}$ ). Table 1 shows the average DOPs with different combinations of the base station height when $N$ is 3 and 4 using absolute range. The digits separated by semicolons in the pair of square brackets are the height ( $z$ value) of the base stations. To make the calculation meaningful, when the value of GDOP at a specific location is too large or the DOP could not be calculated, a value of 1000 is assigned. Similarly, the results of four-sided and five-sided regular polygons for TOA pseudorange processing are presented in Table 2. For TOA pseudorange processing, at least four measurements are required for 3D positioning, so no result for a three-sided polygon is reported. Figures 2 and 3 give four examples of the GDOPs with pseudorange processing and absolute range processing. One can see several interesting things from these results. Firstly as expected, the more transmitters, the better (smaller)
Table 1. The average DOPs for three-sided and four-sided regular polygons with different combinations of base station heights using absolute range measurements (five-sided regular polygon with heights $=[5 ; 5 ; 5 ; 5 ; 5]$ is also listed).

\begin{tabular}{ccccc}
\hline Heights & GDOP & PDOP & HDOP & VDOP \\
\hline$[0 ; 5 ; 0]$ & 44.3 & 44.3 & 9.8 & 44.2 \\
{$[0 ; 5 ; 5]$} & 14.5 & 14.5 & 2.0 & 14.4 \\
{$[5 ; 5 ; 5]$} & 6.1 & 6.1 & 1.4 & 6.0 \\
{$[0 ; 5 ; 0 ; 5]$} & 7.7 & 7.7 & 1.1 & 7.6 \\
{$[0 ; 0 ; 5 ; 5]$} & 28.8 & 28.8 & 8.6 & 28.7 \\
{$[0 ; 0 ; 0 ; 5]$} & 16.5 & 16.5 & 1.3 & 16.5 \\
{$[0 ; 5 ; 5 ; 5]$} & 7.0 & 7.0 & 1.2 & 6.8 \\
{$[0 ; 1.7 ; 3.3 ; 5]$} & 11.1 & 11.1 & 1.3 & 11.0 \\
{$[5 ; 5 ; 5 ; 5]$} & 4.5 & 4.5 & 1.1 & 4.3 \\
{$[5 ; 5 ; 5 ; 5 ; 5]$} & 3.7 & 3.7 & 0.9 & 3.6 \\
\hline
\end{tabular}

Table 2. The average DOPs for four-sided and five-sided polygon with different combinations of base station heights using pseudorange measurement.

\begin{tabular}{cccccc}
\hline Heights & GDOP & PDOP & HDOP & VDOP & TDOP \\
\hline$[0 ; 5 ; 0 ; 5]$ & 11.8 & 11.8 & 1.6 & 11.6 & 1.2 \\
{$[0 ; 0 ; 5 ; 5]$} & 401.1 & 400.6 & 217.5 & 399.6 & 211.9 \\
{$[0 ; 0 ; 0 ; 5]$} & 23.9 & 23.9 & 2.1 & 23.8 & 1.3 \\
{$[0 ; 5 ; 5 ; 5]$} & 34.0 & 33.7 & 7.8 & 33.2 & 7.7 \\
{$[0 ; 1.7 ; 3.3 ; 5]$} & 47.5 & 47.2 & 8.6 & 46.9 & 8.3 \\
{$[5 ; 5 ; 5 ; 5]$} & 210.0 & 208.8 & 92.1 & 208.3 & 99.6 \\
{$[0 ; 5 ; 0 ; 5 ; 5]$} & 10.4 & 10.3 & 1.5 & 10.2 & 1.2 \\
{$[0 ; 5 ; 0 ; 5 ; 0]$} & 10.8 & 10.8 & 1.4 & 10.7 & 0.9 \\
{$[0 ; 0 ; 5 ; 5 ; 0]$} & 26.5 & 26.4 & 2.7 & 26.2 & 1.8 \\
{$[0 ; 0 ; 5 ; 5 ; 5]$} & 31.4 & 31.2 & 4.0 & 30.9 & 3.3 \\
{$[0 ; 0 ; 0 ; 0 ; 5]$} & 17.8 & 17.8 & 1.5 & 17.7 & 0.8 \\
{$[5 ; 5 ; 5 ; 5 ; 0]$} & 16.9 & 16.7 & 2.3 & 16.5 & 2.3 \\
{$[0 ; 1.25 ; 2.5 ; 3.75 ; 5]$} & 25.8 & 25.7 & 2.4 & 25.5 & 2.3 \\
{$[5 ; 5 ; 5 ; 5 ; 5]$} & 113.9 & 113.2 & 31.4 & 112.8 & 36.9 \\
\hline
\end{tabular}

average DOPs can be achieved. Secondly, with similar configurations $(N-1$ base stations with absolute range processing and $N$ base stations with pseudorange processing are regarded a fair comparison pair), absolute range processing tends to provide better DOPs. Thirdly, in absolute range processing, the lowest GDOP and VDOP can be achieved (HDOP is slightly worse than the lowest value) when all the base stations have the same 

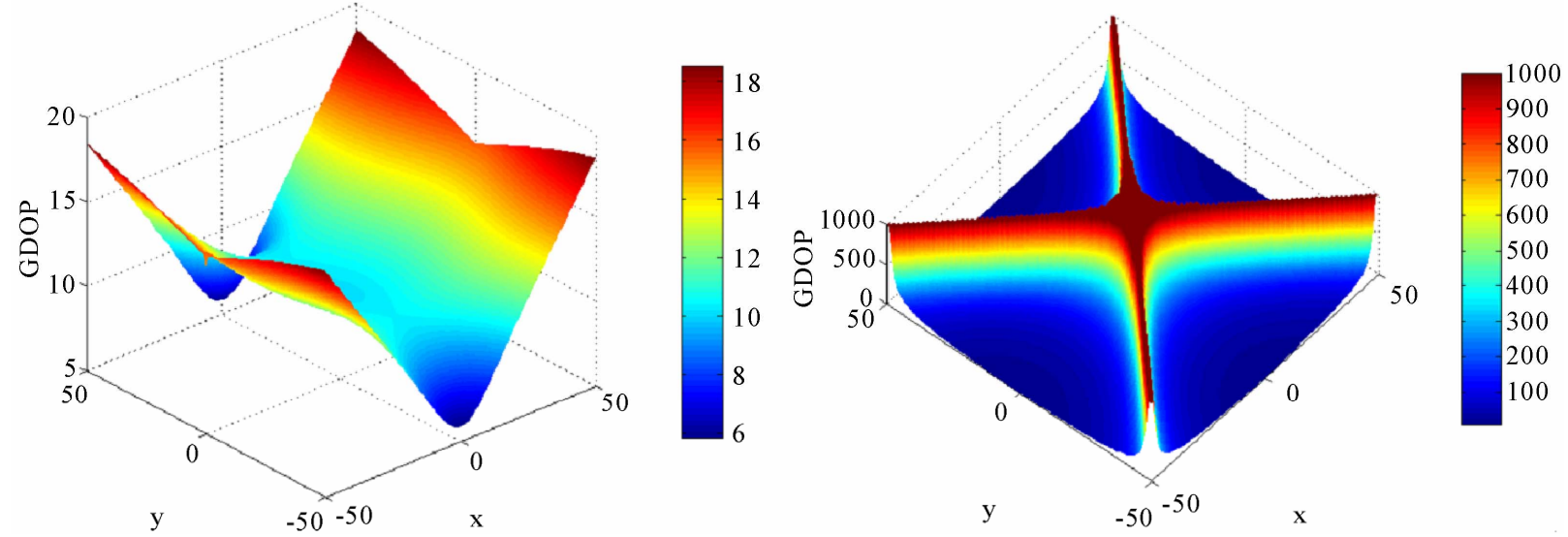

Figure 2. GDOP of Pseudorange processing when $N=4$, height $=[0 ; 5 ; 0 ; 5]$ (top) and [5;5;5;5] (bottom).
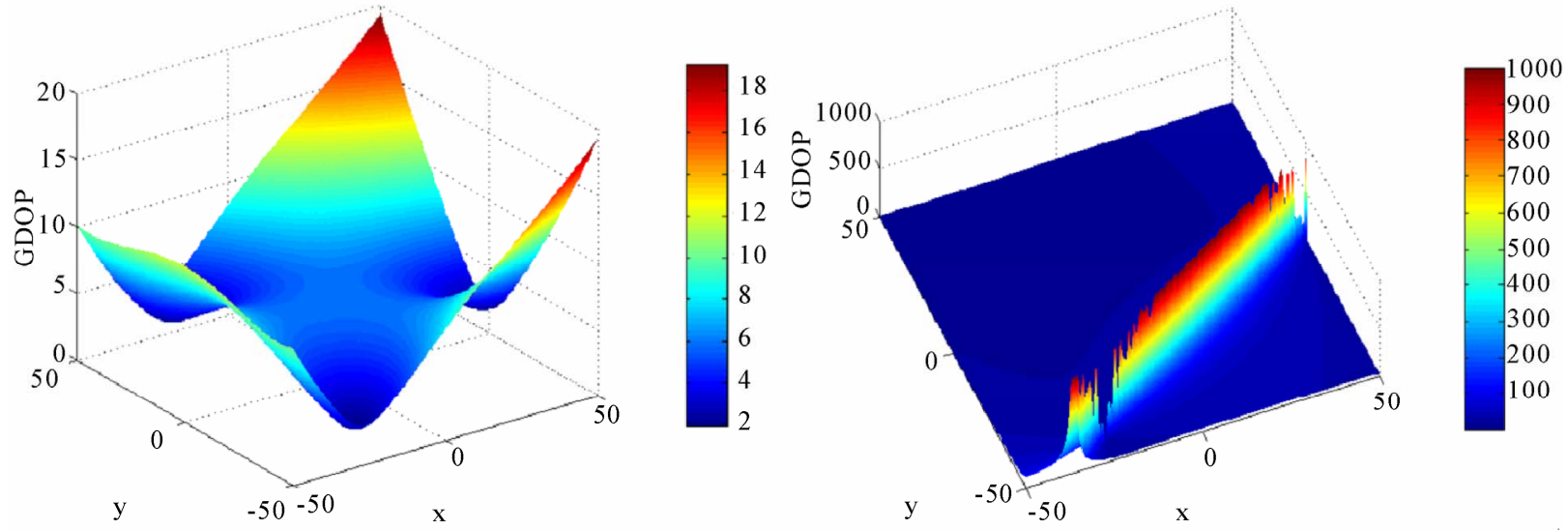

Figure 3. GDOP of absolute range processing when $N=3$, heights $=[0 ; 5 ; 0]$ (top) and $[5 ; 5 ; 5]$ (bottom).

maximum height $(5 \mathrm{~m})$, while in pseudorange processing the base stations should be deployed with minimum and maximum height alternately (e.g. [0;5;0;5] when $N$ is 4). If absolute range processing is possible, clearly it is much better to be utilized. Unfortunately, neither synchronization nor measuring the RTT is a simple task. Finally, VDOP is significantly worse than HDOP. For instance, the best VDOP in the case of four base stations with pseudorange processing, VDOP is 11.6 - 7 times worse than HDOP (1.6). This is expected because of the reduced vertical diversity in base station position. It suggests that for a range measurement error is only 25 $\mathrm{cm}$ (and, considering the poorly behaved indoor environment, $25 \mathrm{~cm}$ error is very small) the minimum $2 \mathrm{D}$ error is as small as $40 \mathrm{~cm}$ which is accurate enough for most applications, but the error in height is $2.9 \mathrm{~m}$, which may locate a fire fighter on the wrong floor of the building.

As the base stations are all deployed on the border of the area of interest, it means the centre of the polygon has a bad DOP. Intuitively, moving one of the base stations to inside the polygon may change the situation. In fact, for pseudorange processing, a three-sided regular polygon with one base station located at $5 \mathrm{~m}(0.1$ times of $a$ ) above the centre improves the DOPs (compared with the four-sided regular polygon). The new GDOP, PDOP, HDOP, VDOP and TDOP are 9.3, 9.3, 1.7, 9.1 and 0.9 respectively (refer to Tables 2 and 3). All the DOPs except HDOP are improved (although not significantly). When $N=4$ (plus one base station in the centre), a similar result can be found. However, in the case of absolute range processing, the DOPs are not improved (see Tables 1 and 3). As pseudorange processing is widely used, the new deployment is of interest. The extra base station can be deployed anywhere inside the polygon. Figure 4 shows clearly that the location above the centre of the polygon generates the best average DOPs. This figure was generated by setting a four-sided regular polygon with a base station which is projected inside the polygon. The height of the transmitter was set to $2 a$. When this transmitter located differently, different DOPs can be obtained. The further the transmitter moves from the centre, the worse the average GDOP can be calculated. 
Table 3. The average DOPs when a transmitter is located in the centre of the polygon.

\begin{tabular}{|c|c|c|c|c|c|c|}
\hline & Heights & GDOP & PDOP & HDOP & VDOP & TDOP \\
\hline \multirow{2}{*}{ Pseudo range } & {$[0 ; 0 ; 0]+5$} & 9.3 & 9.3 & 1.7 & 9.1 & 0.9 \\
\hline & {$[0 ; 0 ; 0 ; 0]+5$} & 8.6 & 8.6 & 1.3 & 8.4 & 0.7 \\
\hline \multirow{2}{*}{ Absolute range } & {$[5 ; 5 ; 5]+5$} & 5.4 & 5.4 & 1.3 & 5.2 & \\
\hline & {$[5 ; 5 ; 5 ; 5]+5$} & 3.9 & 3.9 & 1.0 & 3.8 & \\
\hline
\end{tabular}

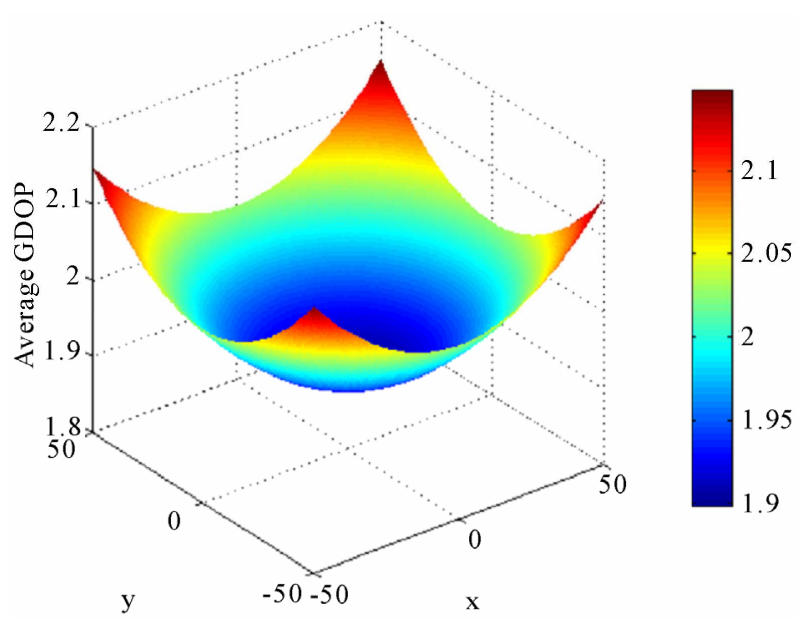

Figure 4. The average GDOP of different locations of the base station inside a four-sided polygon.

If the height of the one in the centre changed, the DOPs may also change. Figure 5 shows when the height of the base station in the centre increases, the HDOP and GDOP decrease dramatically at the beginning (height $<$ $a$ ), then become flat quickly when the height reaches $a$. VDOP and TDOP are always the same (presented as a flat line). Obviously, to achieve a good VDOP, the height should have a similar magnitude to the range between the base stations on the $x-y$ plane (e.g. $a$ ). This requirement sometimes is hard to meet. Using the fire fighter's example again, it is impossible to place a base station above field of fire, especially about $50 \mathrm{~m}$ high from the ground. Hence the height estimation of the user is always significant worse than the $2 \mathrm{D}$ coordinates. If a more accurate height is needed, alternative methods such as a barometer [21] must be considered. Where alternatives are not possible, the deployment of the base stations is restricted by the height of the transmitters above the ground - that is the transmitters on the ground (or close to the ground) cannot be significantly separated.

\section{Concluding Remarks}

DOP is an important factor for positining when range or angle measurement is used. This paper investigates the DOPs in $3 \mathrm{D}$ positioning applications using range meas-

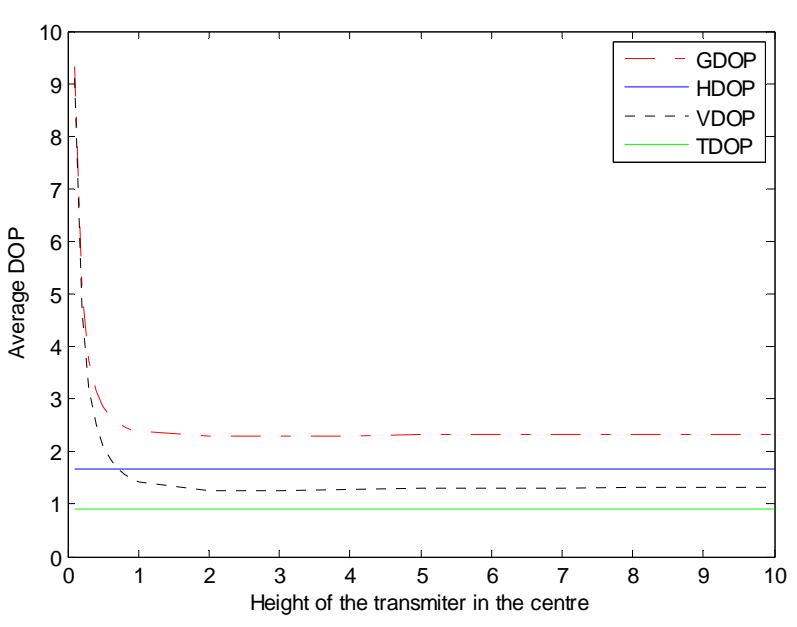

Figure 5. The average DOPs of the three-sided regular polygon with one base station in the centre (the height unit is $a$ ).

urements. The performance of two types of measurements-absolute range and pseudorange has been discussed.

It has been found that to achieve the best (lowest) average GDOP, the deployment of the base stations for absolute range and pseudorange processing are different. In the case of the $N$ points located at the vertices of a $N$-sided regular polygon, the transmitters should be deployed with minimum and maximum height alternatively for pseudorange processing and with the same maximum height (the maximum height can be up to a, if it is larger than a, a should be chosen) for absolute range processing. Changing the setup from $N$-sided regular polygon to $(N-$ 1)-sided polygon plus one station located in the centre with maximum height decreases the DOPs for pseudorange processing.

General speaking, using absolute range processing requires fewer transmitters and can guarantee lower DOPs. However, to make absolute measurements is not a simple task. Pseudorange processing is still most widely used in GNSS. For pseudorange processing, putting a base station above the area of interest can lead to better DOPs. But for some applications, it is impossible to deploy transmitters in that way, especially because the magnitude of the height is significant. Alternative methods must 
be chosen to avoid the much lower accuracy in height. When TDOA is used, the conclusions drawn from TOA pseudorange are also true.

\section{References}

[1] B. W. Parkinson and J. J. Spilker, "Global Positioning System: Theory an Applications, Volume I," American Institute of Aeronautics and Astronautics, Inc., Washington, 1996.

[2] C. Rizos, G. Roberts, J. Barnes and N. Gambale, "Experimental Results of Locata: A High Accuracy Indoor Positioning System," 2010 International Conference on Indoor Positioning and Indoor Navigation (IPIN), Zurich, 15-17 September 2010, pp. 1-7. doi:10.1109/IPIN.2010.5647717

[3] D. Cyganski, J. Duckworth, S. Makarov, W. Michalson, J Orr, V. Amendolare, J. Coyne, H. Daempfling, S. Kulkarni and H. Parikh, "WPI Precision Personnel Locator System," Proceeding Institute of Navigation, National Technical Meeting, San Diego, 22-24 January 2007, p. 22.

[4] N. B. Priyantha, A. Chakraborty and H. Balakrishnan, "The Cricket Location-Support System," Proceedings of the 6th Annual International Conference on Mobile Computing and Networking ACM, New York, 6-11 August, 2000, p. 32.

[5] M. Rabinowitz, and J. J. Spilker Jr, “A New Positioning System Using Television Synchronization Signals," IEEE Transactions on Broadcasting, Vol. 51, No. 1, 2005, pp. 51-61. doi:10.1109/TBC.2004.837876

[6] T. Sathyan, D. Humphrey, M. Hedley and M. Johnson, "A Wireless Indoor Localization Network-System Introduction and Trial Results," IGNSS Symposium 2009, Gold Coast, 1-3 December.

[7] T. G. Thorne (Ed.), "Navigation Systems for Aircraft and Space Vehicles," Pergamon Press, Oxford, 1962.

[8] C. Drane, M. Macnaughtan and C. Scott, "Positioning GSM Telephones," IEEE Communications Magazine, Vol. 36, No. 4, 1998, pp. 46-54. doi:10.1109/35.667413

[9] B. Hofmann-Wellenhof, H. Lichtenegger and J. Collins, "GPS Theory and Practice," 5th Edition, Springer, New York, 2001.

[10] E. Kaplan (Ed.), "Understanding GPS: Principles and Applications," 2nd Edition, Artech House, Norwood, 2005.
[11] R. Yarlagadda, I. Ali, N. Al-Dhahir and J. Hershey, "GPS GDOP Metric," IEE Proceedings Radar Sonar and Navigation, Vol. 147, No. 5, 2002, pp. 259-264. doi:10.1049/ip-rsn:20000554

[12] R. B. Langley, "Dilution of Precision," GPS World, Vol. 10, No. 5, 1999, pp. 52-59.

[13] H. B. Lee, "Accuracy Limitations of Hyperbolic Multilateration Systems," IEEE Transactions on Aerospace and Electronic Systems, Vol. AES-11, No. 1, 1975, pp. 16-29. doi:10.1109/TAES.1975.308024

[14] H. B. Lee, "A Novel Procedure for Assessing the Accuracy of Hyperbolic Multilateration Systems," IEEE Transactions on Aerospace and Electronic Systems, Vol. AES-11, No. 1, 1975, pp. 2-15. doi:10.1109/TAES.1975.308023

[15] Y. Zhao, "Mobile Phone Location Determination and Its Impact on Intelligent Transportation Systems," IEEE Transactions on Intelligent Transportation Systems, Vol. 1, No. 1, 2000, p. 55. doi:10.1109/6979.869021

[16] H. Laitinen, S. Ahonen, S. Kyriazakos, J. Lahteenmaki, R. Menolascino and S. Parkkila, "Cellular Location Technology," 2000. http://www.vtt.fi/tte/tte35/pdfs/CELLOWP2-VTT-D03-0 07-Int.pdf

[17] P. Steggles and S. Gschwind, "The Ubisense Smart Space Platform," Adjunct Proceedings of the Third International Conference on Pervasive Computing, Vol. 191, May 2005, pp. 73-76.

[18] A. G. Dempster, "Dilution of Precision in Angle-of-Arrival Positioning Systems," Electronics Letters, Vol. 42, No. 5, 2006, pp. 291-292. doi:10.1049/el:20064410

[19] N. Levanon, "Lowest GDOP in 2-D Scenarios," IEE Proceedings-Radar, Sonar and Navigation, Vol. 147, No. 3, 2002, pp. 149-155. doi:10.1049/ip-rsn:20000322

[20] D. H. Shin and T. K. Sung, "Comparisons of Error Characteristics between TOA and TDOA Positioning," IEEE Transactions on Aerospace and Electronic Systems, Vol. 38, No. 1, 2002, pp. 307-311. doi:10.1109/7.993253

[21] Vaisala, "PTU300 Combined Pressure, Humidity and Temperature Transmitter for Demanding Applications," 2011.

http://www.vaisala.com/Vaisala\%20Documents/Brochure s\%20and\%20Datasheets/PTU300-Combined-DatasheetB210954EN-B-LoRes.pdf 